\title{
Nonlinear Froude-Krylov and viscous drag representations for wave energy converters in the computation/fidelity continuum
}

\author{
Giuseppe Giorgi*, John V. Ringwood \\ Center for Ocean Energy Research, Maynooth University, Maynooth, Co. Kildare, Ireland
}

\section{A R T I C L E I N F O}

\section{Keywords:}

Nonlinear hydrodynamic modelling

Nonlinear Froude-Krylov force

Viscous drag

Computational fluid dynamics

Fidelity

Computational time

\begin{abstract}
A B S T R A C T
Designing, optimizing and controlling a wave energy converter requires the construction of a mathematical model in order to simulate the behaviour of the device. Given the nonlinear nature of fluid-structure interactions, the definition of the model is not straightforward and should take into account the specific application it is intended for. Two of the most important characteristics of a model are the computational time and the expected accuracy, which usually are mutually conflicting. The inclusion of nonlinearities potentially increases the model accuracy, but at a higher computational price.

Considering a heaving wave energy converter with and without the application of latching control, this paper studies and compares nine different modelling options, eight of which are based on potential theory and consider nonlinear Froude-Krylov and viscous drag forces, while one is based on fully-nonlinear computational fluid dynamics. The value of including nonlinearities in the hydrodynamic model is discussed in relation to the computational cost of the eventual accuracy benefits, under a range of scenarios.
\end{abstract}

\section{Introduction}

Mathematical models for wave energy converters (WECs) are an essential numerical tool used for device design, optimization, control and management. Numerous possible approaches to modelling are available, as shown in Penalba et al. (2015), and the choice of an appropriate model needs to take into account specific requirements demanded by the intended application of the model itself. In particular, two of the most important characteristics of a model are the computational time required by the calculations and the expected accuracy of the results. Such characteristics are generally conflicting; therefore, an appropriate compromise has to be defined. In particular, a higher accuracy of the results can be obtained by including further nonlinearities in the model, inexorably increasing, to some extent, the model complexity and computational time.

For preliminary studies, such as early stage concept development or approximate array calculations, low accuracy may be acceptable, while a fast computation is mandatory. Fully-linear models are therefore widely used, since they can return reasonable results rapidly. Conversely, for survivability, extreme events, and loads studies, accurate results can be returned only by very complex and time consuming models such as, for example, fully-nonlinear CFD (computational fluid dynamics) models.

In between, for power production assessment and model-based controller tuning and optimization, medium/high fidelity, with an acceptable computation time, is required to correctly define the parameters of the system and the predicted performance of the device, over a wide range of sea conditions. Furthermore, mathematical models for control applications must compute quickly enough to allow the control strategy to take action in real time.

This paper focuses on the power production region of a small heaving sphere, operating under the application of a latching control strategy. Note that, in the unlikely situation that no control is implemented, linear models are normally quite accurate for small heaving WECs, which are characterized by a high and peaky resonance frequency: usually operating in sea states at frequency lower than resonance, such devices behave, in absence of a control strategy, as wave followers, and nonlinear models are not likely to produce any significant advantage, as shown in Giorgi et al. (2016b). Nevertheless, the purpose of real-world deployed WECs is to maximize power capture; such an objective is pursued by the control strategy. Commonly, control strategies, such as latching control, seek the objective of increasing power absorption by increasing the motions amplitude and, consequently, the relevance of nonlinearities. When such control strategies are employed, it is expected that nonlinear models are able to considerably improve the fidelity of the model fit, at an additional computational cost, which depends on the complexity of the specific nonlinear model (Giorgi et al., 2016b).

\footnotetext{
* Corresponding author.

E-mail address: giuseppe.giorgi.2015@mumail.ie (G. Giorgi).
} 
This paper seeks to evaluate the performance of a wide range of nonlinear models, relevant to heaving WECs, in terms of accuracy and computational cost. In total, nine different implementations of prominent nonlinearities in the equations of motion are considered; while one model is based on fully-nonlinear CFD software, the remaining eight models are based on different implementations of Froude-Krylov (FK) and viscous drag forces (four FK force modelling options multiplied by two viscous drag modelling options). Note that, notwithstanding such modelling approaches are different implementations of the same equation of motion, they are referred to as different nonlinear models in order to make the discussion and comparison more clear. Since CFD is fully-nonlinear, CFD results are taken as a "gold standard" benchmark, in order to validate the other models and compare their accuracy.

The reminder of the paper is organized as follows: Section 2 presents the details of the hydrodynamic models considered in this paper. A case study is presented in Section 3 and results are shown in Section 4. Finally, some discussion and conclusions are presented, respectively, in Sections 5 and 6.

\section{Hydrodynamic models}

Eight of the nine hydrodynamic models have their foundations in potential theory (PoT), described in Section 2.1. Different options, for modelling of FK and viscous drag forces, described respectively in Sections 2.1.1 and 2.1.2, discriminate between each of the models considered here. A summary of the different models is presented in Table 1. On the other hand, the last model is based on the application of the Navier-Stokes equations (CFD), which are described in Section 2.2 .

\subsection{Potential theory-based (PoT) models}

Under the assumption of inviscid fluid, and irrotational and incompressible incident flow, the dynamics of a floating system can be described by Newton's second law:

$m \ddot{\mathbf{z}}(t)=\mathbf{F}_{g}-\iint_{S(t)} P(t) \mathbf{n} d S+\mathbf{F}_{P T O}(t)$

where $m$ is the mass of the floater, $\mathbf{z}$ the vertical displacement of the body from its hydrostatic equilibrium position, $\mathbf{F}_{g}$ the gravity force, $S(t)$ the submerged wetted surface, $P$ the pressure, $\mathbf{n}$ the vector normal to the surface, and $\mathbf{F}_{P T O}$ the power take-off (PTO) force.

Applying Bernoulli's equation (Newman, 1977) to the incident flow, the formulation of the pressure $P$ is obtained as:

$P(t)=-\rho g z(t)-\rho \frac{\partial \phi(t)}{\partial t}-\rho \frac{|\nabla \phi(t)|^{2}}{2}$

where $\rho$ is the density of water, $g$ the acceleration due to gravity, $P_{s t}=-\rho g z$ the hydrostatic pressure, and $\phi$ the potential flow which, based on linear wave theory, is the sum of the undisturbed incident

\section{Table 1}

Summary of the 8 potential theory-based (PoT) models considered in this paper, where $\bigcirc$ and $\bullet$ stand, respectively, for linear and nonlinear representation of the static FK force $\left(F_{F K_{S t}}\right)$ and dynamic FK force $\left(F_{F K_{d y}}\right)$, and absence/presence of the viscous drag force $\left(F_{V i s}\right)$, as defined in Section 2.

\begin{tabular}{lllll}
\hline & PoT models & $F_{F K_{S t}}$ & $F_{F K_{d y}}$ & $F_{v i s}$ \\
\hline 1 & LFKnoD & 0 & 0 & 0 \\
2 & NLRnoD & $\bullet$ & 0 & 0 \\
3 & NLFKanoD & $\bullet$ & $\bullet$ & 0 \\
4 & NLFKrnoD & $\bullet$ & $\bullet$ & 0 \\
5 & LFKD & 0 & 0 & $\bullet$ \\
6 & NLRD & $\bullet$ & 0 & $\bullet$ \\
7 & NLFKaD & $\bullet$ & $\bullet$ & $\bullet$ \\
8 & NLFKrD & $\bullet$ & $\bullet$ & \\
\hline
\end{tabular}

flow potential $\phi_{I}$, the diffraction potential $\phi_{D}$, and the radiation potential $\phi_{R}$ :

$\phi(t)=\phi_{I}(t)+\phi_{D}(t)+\phi_{R}(t)$

Therefore, three time-varying pressures, namely incident pressure $P_{I}$, diffraction pressure $P_{D}$, and radiation pressure $P_{R}$, can be defined by substituting the potentials $\phi_{I}, \phi_{D}$, and $\phi_{R}$ in Eq. (3) into Bernoulli's Eq. (2).

Combining Eqs. (1)-(3), different forces can be defined:

- $\vec{F}_{F K_{s t}}$ is the static Froude-Krylov force, given as the balance between the gravity force and the Archimedes force:

$\vec{F}_{F K_{S t}}(t)=\overrightarrow{F_{g}}-\iint_{S(t)} P_{s t}(t) \vec{n} d S$

- $\vec{F}_{F K_{d y}}$ is the dynamic Froude-Krylov force:

$\vec{F}_{F K_{d y}}(t)=-\iint_{S(t)} P_{I}(t) \vec{n} d S$

- $\overrightarrow{F_{D}}$ is the diffraction force:

$$
\overrightarrow{F_{D}}(t)=-\iint_{S(t)} P_{D}(t) \vec{n} d S
$$

- $\overrightarrow{F_{R}}$ is the radiation force:

$$
\overrightarrow{F_{R}}(t)=-\iint_{S(t)} P_{R}(t) \vec{n} d S
$$

It is now possible to rewrite Eq. (1) as:

$m \ddot{\mathbf{z}}=\mathbf{F}_{F K_{S t}}+\mathbf{F}_{F K_{d y}}+\mathbf{F}_{D}+\mathbf{F}_{R}+\mathbf{F}_{P T O}$

Possible sources of nonlinearity in Eqs. (1)-(8) are the quadratic terms, the eventually nonlinear incident potential flow in Bernoulli's Eq. (2), and the instantaneous variation of the wetted surface $S(t)$ in Eq. (1). The quadratic terms in Eq. (2) can be neglected for small heaving WECs, as shown in Merigaud et al. (2012), as only linear waves are considered, which cover the vast majority of the waves in the power production region.

Radiation and diffraction forces are assumed linear; such an assumption, not directly verified in this study, is reasonable for devices much smaller than the wavelength (Falnes et al., 2002). Furthermore, Giorgi et al. (2016a) shows that radiation and diffraction forces are much smaller than FK forces, and so are less relevant in altering the dynamics of the body. Therefore, considering the mean wetted surface, diffraction forces are computed by means of a convolution product between the free surface elevation and the diffraction impulse response function, while a state space representation is used to compute radiation forces (Taghipour et al., 2007).

Different approaches for FK force modelling are proposed in Section 2.1.1, considering (or not) the instantaneous wetted surface for the static and dynamic pressure integrals. Progressively, Section 2.1.2 deals with the eventual inclusion of viscous effects in the dynamical equation of the system.

Each one of the eight resulting models will be labeled by two indeces: the first one refers to the FK force modelling mode, namely linear FK (LFK), nonlinear restoring (NLR), re-meshing nonlinear FK (NLFKr) and algebraic nonlinear FK (NLFKa); the second one indicates the absence (noD) or the inclusion (D) of a viscous drag term.

Note that improving the accuracy of the mathematical description of the different components of the PoT model, by means of different implementations of FK force models and eventually including viscous drag effects, does not necessarily produce more accurate results. In 
fact, given the number of force components in Eq. (8), different modelling errors sources are present, which may add up (increasing the total error), or subtract (decreasing the total error). When two (or more) modelling errors partially cancel out, a "mitigation" effect is achieved, as described in Section 2.1.1.

\subsubsection{Froude-Krylov force modelling}

Differences between the proposed modelling options for FK force representation consider how the instantaneous wetted surface is, or is not, taken into account in the calculation of the static and dynamic FK forces. Therefore, differences in the computed force depend on the relative displacement $\left(z_{r e l}\right)$ between the device position $\left(z_{d}\right)$ and the free surface elevation $(\eta)$. In particular, a significantly different behaviour can be observed in the extreme (unrealistic) situation when the relative displacement is larger than the physical dimension of the device, namely when the body is clear of the water surface. Such a situation is not uncommon, when fully-linear models of heaving WECs under latching control are considered, as shown in Giorgi et al. (2016b). Given the absence of any fluid-structure interaction, the physically correct answer when the body is completely out of the water would be a restoring force equal to the weight of the device (independent of the body position) and a null excitation force.

Linear Froude-Krylov force (LFK). According to the fully-linear representation of FK forces, the mean wetted surface is considered for both static, and dynamic, pressure integrals. Therefore, the hydrostatic stiffness $K_{h}$ is used, proportional to the cross-sectional area at the still water level $\left(A_{S W L}\right)$ :

$\mathbf{F}_{F K_{S t}}=-K_{h} z=-\rho g A_{S W L} z$

Note that such a restoring force assumes the device to be an infinitely long vertical prism, namely with constant cross sectional area and always piercing the water. Consequently, the restoring force linearly increases with displacement, irrespective of the actual change in body form and cross sectional area.

The excitation impulse response function is used for the calculation of the dynamic FK and diffraction force; therefore, the excitation force is calculated with reference to the mean wetted surface, irrespective of the body position. Consequently, when the body is completely out of the water, the excitation force continues pushing the body but, at the same time, the restoring force is linearly increasing with the displacement, so the two modelling errors counteract and a "mitigation" effect is achieved.

Nonlinear restoring force (NLR). For the nonlinear restoring force model (NLR), as shown in Fig. 1, the integral of the static pressure is computed over the instantaneous wetted surface $S(t)$, which can be described as the closed surface $S_{c}$ minus the horizontal surface $S_{W P}$, as shown in Forehand et al. (2016). $S_{W P}$ is defined as the intersection between the body and the horizontal plane at a free surface elevation $\eta$.
Consequently, $\mathbf{F}_{F K_{s t}}$ can be computed as follows:

$\mathbf{F}_{F K_{s t}}=\mathbf{F}_{g}-\left(\iint_{S_{c}} P_{s t} \mathbf{n} d S-\iint_{S_{W P}} P_{s t} \mathbf{n} d S\right)$

Applying Gauss's divergence theorem to the integral over the closed surface $S_{c}$, Eq. (10) becomes:

$\mathbf{F}_{F K_{s t}}=\mathbf{F}_{g}+\left(\rho g V_{s u b}-\rho g \eta A_{W P}\right) \mathbf{k}$

where $V_{\text {sub }}$ is the submerged volume enclosed by $S_{c}, A_{W P}$ is the area of $S_{W P}$, and $\mathbf{k}$ is the vertical unity vector.

The nonlinear restoring force is smaller than the linear one in cases such as a sphere, where $A_{W P}$ is smaller than $A_{S W L}$, and reaches a plateau when the body is completely out of the water. However the excitation force is linear; therefore, there is no "mitigation" effect, and amplitudes larger than the linear model are expected.

Nonlinear Froude-Krylov forces (NLFK). For the nonlinear FK models (NLFK), the FK force integrals are solved over the instantaneous wetted surface. Static and dynamic pressures are collectively obtained by applying Airy's wave theory for deep water waves:

$P(x, z, t)=\rho g a \mathrm{e}^{\chi z} \cos (\omega t-\chi x)-\rho g z$

where $x$ is the direction of wave propagation, $a$ is the wave amplitude, $\chi$ the wave number, and $\omega$ the wave frequency.

Generally, such FK force integrals have to be solved numerically. The geometry is discretized into small plane panels, allowing the computation of FK forces for shapes of arbitrary complexity. The recalculation of the instantaneous wetted surface requires a computationally expensive re-meshing routine, which redefines the panels each time step, as the free surface elevation changes. A detailed description of the re-meshing approach is given in Gilloteaux (2007). Methods requiring a re-meshing routine will be hereafter referred to as NLFKr.

In the case where the device is an axisymmetric heaving device, such as the one shown in Fig. 2, an algebraic solution to the FK force integrals is achievable (NLFKa), as shown in Giorgi and Ringwood (2016b).

The axisymmetric geometry makes possible the use of parametric cylindrical coordinates $[\sigma, \theta]$ to describe the surface of the body:

$\left\{\begin{array}{l}x(\sigma, \theta)=f(\sigma) \cos \theta \\ y(\sigma, \theta)=f(\sigma) \sin \theta, \quad \sigma \in\left[\sigma_{1}, \sigma_{2}\right] \wedge \theta \in[0,2 \pi) \\ z(\sigma, \theta)=\sigma\end{array}\right.$

Referring to the notation of Fig. 2, the resulting FK force in heave is computed as follows:

$\mathbf{F}_{F K_{z}}=\mathbf{F}_{g}-\int_{0}^{2 \pi} \int_{\sigma_{1}}^{\sigma_{2}} P(x(\sigma, \theta), z(\sigma, \theta), t) f^{\prime}(\sigma) f(\sigma) \mathbf{k} d \sigma d \theta$

where the limits of integration, which define the instantaneous wetted

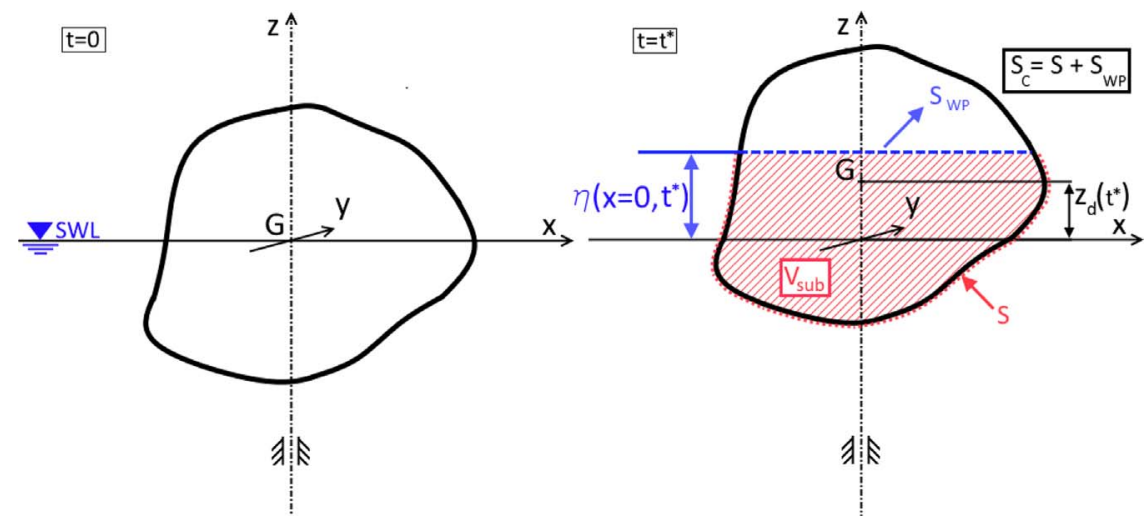

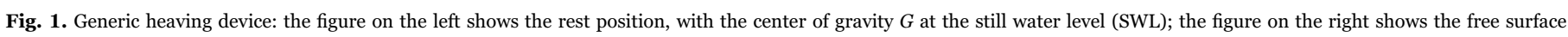

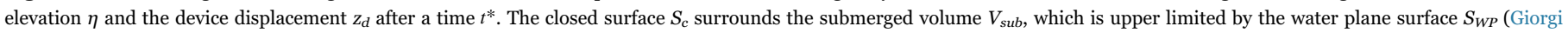
et al., 2016b). 


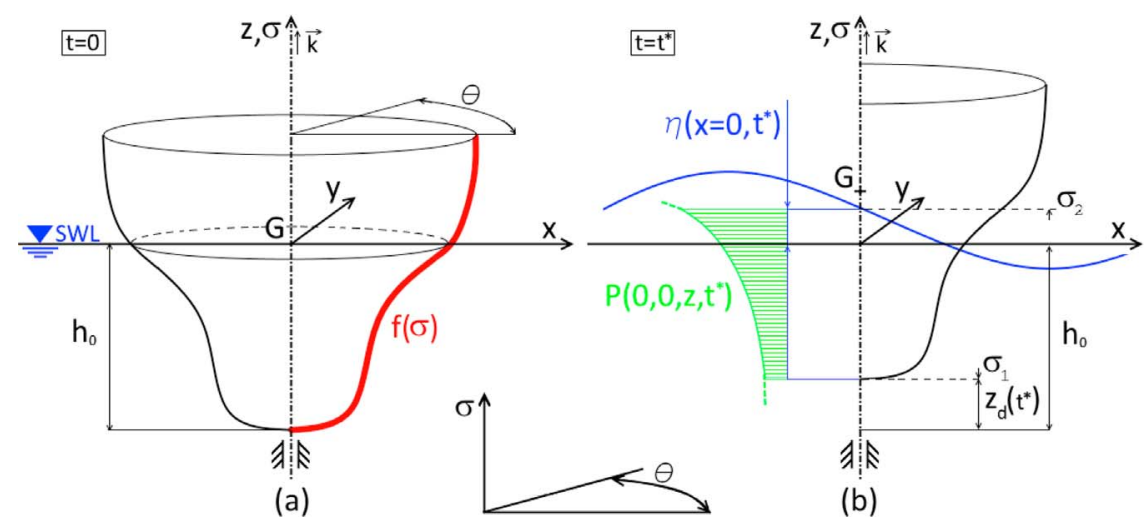

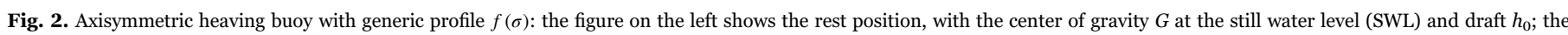

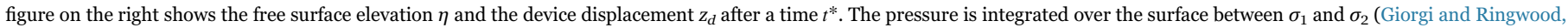
2016b).

surface, are $\sigma_{1}=z_{d}-h_{0}$ and $\sigma_{2}=\eta$.

Note that, in case of an axisymmetric heaving device, the remeshing (NLFKr) and algebraic (NLFKa) approaches return identical results, albeit at a different computational cost (Giorgi and Ringwood, 2016b).

\subsubsection{Viscous drag modelling}

One assumption of potential flow theory is that the fluid is inviscid; therefore, viscous effects are neglected. One common way of including viscous drag effects in the equation of motion is by means of a Morisonlike term (Bhinder et al., 2011). The Morison equation $F_{m o r}$ describes the total force on a fixed body in an oscillatory flow (Morison et al., 1950):

$\mathbf{F}_{\text {mor }}=-\frac{1}{2} \rho C_{d} A_{d}\left|\mathbf{V}_{0}\right| \mathbf{V}_{0}-\rho C_{M} V_{d} \dot{\mathbf{V}}_{0}$,

where $C_{d}$ is the drag coefficient, $C_{M}$ is the inertia coefficient, $A_{d}$ is the characteristic area, $\mathbf{V}$ is the velocity of the floater, $\mathbf{V}_{0}$ is the undisturbed flow velocity, and $V_{d}$ the volume of displaced fluid.

Viscous forces for a moving wave energy converter can be modelled using only the first term of Eq. (15), considering the relative velocity between the velocity of the floater $\mathbf{V}$ and the undisturbed flow velocity (Bhinder et al., 2011):

$\mathbf{F}_{V i s}=-\frac{1}{2} \rho C_{d} A_{d}\left|\mathbf{V}-\mathbf{V}_{0}\right|\left(\mathbf{V}-\mathbf{V}_{0}\right)$,

Note that the characteristic area is the projection of the instantaneous wetted surface onto a plane normal to the flow. Consequently, the viscous force is not symmetric since, when the body is completely out of the water, $A_{d}$ and the viscous force are null whereas, when the body is fully submerged, $A_{d}$ is at its maximum.

Notwithstanding the simplicity of the viscous force model in (16), the definition of the drag coefficient $C_{d}$ represents a challenge. Ideally, specific experimental tests can be performed in order to identify $C_{d}$, as in Lok et al. (2014). However, accessing wave tank facilities is often not a feasible option, due to high costs of experimental testing. Furthermore, a prototype of the device needs to be available for testing. Such problems may be overcome by performing the identification of $C_{d}$ in a numerical wave tank, using CFD simulations, as in Bhinder et al. (2011).

On the other hand, when simple geometries are considered, it is possible to define $C_{d}$ a priori based on data available in the literature. In the case of heaving floating buoys, the dimensionless KeuleganCarpenter number $\mathrm{KC}$ can be used, which is the ratio between the drag and inertia forces acting on a body in an oscillatory fluid flow (Keulegan and Carpenter, 1956). In the case of sinusoidal motion, the KC number can be computed as:
$K C=2 \pi \frac{A}{L_{c}}$

where $A$ is the amplitude of motion and $L_{c}$ is a characteristic length scale, for example the diameter for a sphere. A further useful dimensionless number is the Reynolds number (Molin, 2002), defined as:

$R e=\frac{\nu L_{c}}{\nu}$

where $v$ is the undisturbed fluid velocity, and $v$ is the kinematic viscosity. Finally, for a particular geometry, experimental graphs can be found in the literature, such as Molin (2002), plotting $C_{d}$ in function of $K C$ and $R e$.

\subsection{Computational fluid dynamics (CFD) model}

The fully-nonlinear model is implemented in a numerical wave tank (NWT) using the open-source CFD software OpenFOAM (OpenFOAM, 2015). The fluid dynamics are described by a set of differential equations, known as the Navier-Stokes equations (Temam, 2001):

$\nabla \cdot \mathbf{u}=0$

$\rho \frac{\partial \mathbf{u}}{\partial t}+\rho \mathbf{u} \cdot \nabla \mathbf{u}=-\nabla P+\nabla \cdot \mathbf{T}+\rho \mathbf{g}$,

where $\mathbf{u}$ is the fluid velocity and $\mathbf{T}$ is the stress deviator, given by.

$\mathbf{T}=\mu\left[\nabla \mathbf{u}+(\nabla \mathbf{u})^{T}\right]$

while $\mu$ is the dynamic viscosity, under the incompressibility assumption $\partial \rho / \partial t=0$.

Full details concerning the definition of the computational domain, and the boundary conditions, are given in Giorgi and Ringwood (2016a). Of particular importance is the detail with which turbulence phenomena are modelled, since several different approaches are available (Ferziger et al., 1997). In this study, $k-\epsilon$ Reynolds averaged Navier-Stokes ( $k-\epsilon$ RANS) simulations are employed, which first solve for the Reynolds-average of Eqs. (19)-(21), and then apply $k-\epsilon$ wall functions in order to take fluctuations due to turbulence into account (Ferziger et al., 1997).

Very high fidelity is expected from this modelling approach, since all nonlinear effects are included. Consequently, results obtained through CFD simulation will be taken in this study as a fidelity benchmark to evaluate the results obtained with the other models.

However, drawbacks of the CFD model are its complexity and computational speed. Besides a computational time several orders of magnitude higher than PoT models, correct design of the NWT dimensions, mesh structure and parameters requires significant effort and experience, often relying on an exhaustive trial and error process. 


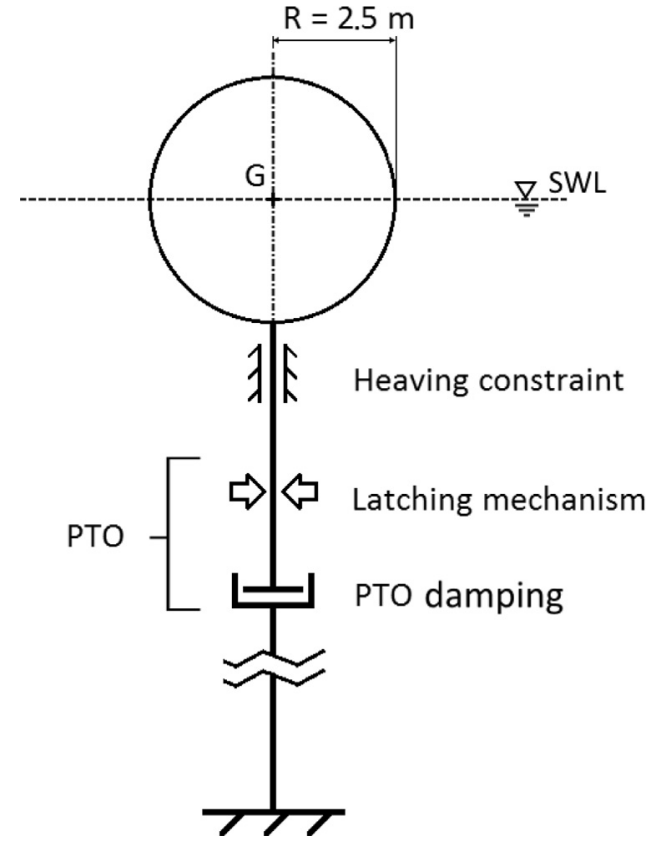

Fig. 3. Case study: a sphere with radius $R$ and with its center of gravity $G$ at the SWL and a cone with draft and radius at the SWL, with $R_{0}=R$. The device is constrained to move in heave only and deep water conditions are assumed. The PTO system is composed of a linear damper and a latching mechanism.

\section{Case study}

The models presented in Section 2 are used to simulate the response of a heaving sphere in deep water conditions, as shown in Fig. 3. The geometry of the body is chosen as a sphere, since cylinders present linear static FK forces, as shown in (9) and discussed in Section 2.1.1 Furthermore, Penalba et al. (2015) shows that significant nonlinearities in FK forces are mainly excited by non-uniform cross sectional area in the direction of motion. The diameter of the sphere is chosen as $5 \mathrm{~m}$, comparable to the floater of the existing Wavestar device (Wavestar, 2016).

The hydrodynamic coefficients have been calculated using the boundary element method (BEM) solver WAMIT (W. Inc, 2013). In order to avoid significant error, and achieving high accuracy in the definition of the hydrodynamic coefficients, a very large number of both panels (1600), and wave frequencies $w$ (600, equally spaced, with a $0.03 \mathrm{rad} / \mathrm{s}$ step) have been used. As a comparison, note that Babarit et al. (2012) performs a convergence study for the very same geometry (half submerged sphere of $5 \mathrm{~m}$ diameter), using 140 and 300 panels. The resulting added mass and radiation damping coefficients are shown in Fig. 4.

A second order Runge-Kutta method with constant time step

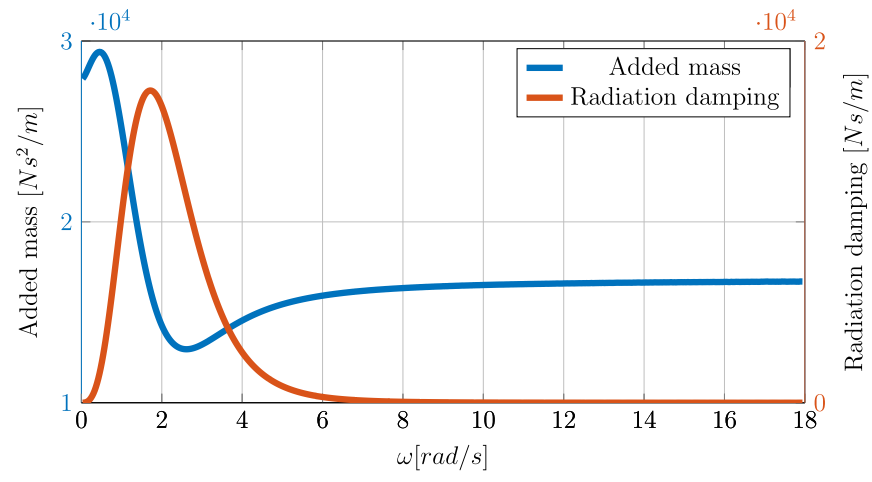

Fig. 4. Added mass and radiation damping for the floating sphere in Fig. 3, using the boundary element method solver WAMIT (W. Inc, 2013).

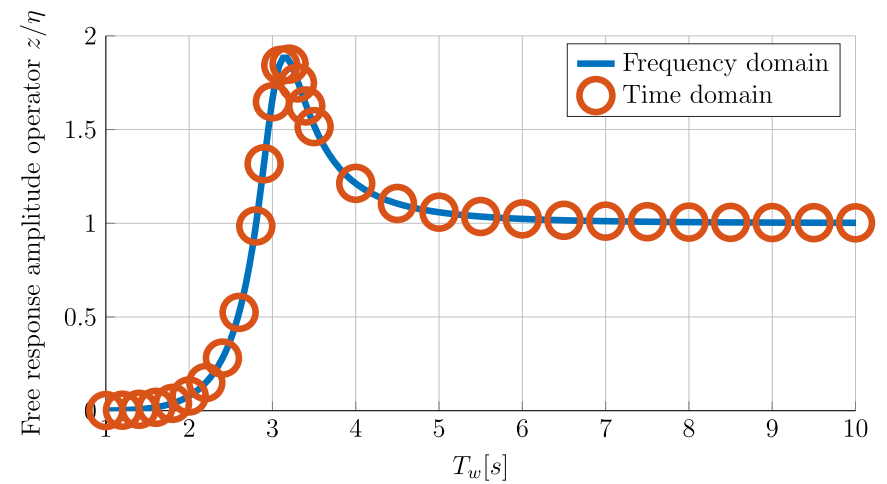

Fig. 5. Free response amplitude operator for the floating sphere in Fig. 3, computed both in the frequency (benchmark) and time domain, with $B_{P T O}$ equal to zero. After a convergence study in the time domain, the time step is chosen equal to $0.01 \mathrm{~s}$, and the two RAOs are significantly overlapping.

$(0.01 \mathrm{~s})$ is used to solve the equation of motion (8) in the time domain. The time step is chosen following a convergence study (using several time steps between $0.2 \mathrm{~s}$ and $0.001 \mathrm{~s}$ ) based on the free response amplitude operator (RAO) which, in the time domain, is defined as the ratio between the simulated displacement of the device and free surface elevation, with $B_{P T O}$ equal to zero. The RAO, computed in the frequency domain, according to W. Inc (2013), is used as a benchmark for the convergence; Fig. 5 shows the resulting RAO, computed both in the time and frequency domain.

The amplitude of motion of the floater in Fig. 3 is likely to be of the same order of magnitude as the characteristic length (the diameter) of the device, resulting in a KC number of about $2 \pi$ and, according to Molin (2002) and Babarit et al. (2012), a drag coefficient $C_{d}$ of unity.

A set of regular waves is considered, in order to analyze the frequency and amplitude effects independently. 24 wave conditions are considered, whose heights range from $0.5 \mathrm{~m}$ to $2 \mathrm{~m}$, with a $0.5 \mathrm{~m}$ step, and periods ranging from $5 \mathrm{~s}$ to $10 \mathrm{~s}$, with a $1 \mathrm{~s}$ step.

The free surface elevation in the NWT is generated through waves2Foam (Jacobsen et al., 2012), which implements numerical generation and absorption zones, which gradually blend the required wave profile into the numerical domain. Consequently, the actual wave profile obtained depends on many factors, among which the most important are the length of the domain, and the size of the mesh. Therefore, the free surface elevation at the position of the body is measured by running a simulation without the body using a twodimensional section of the NWT. In practice, the measured free surface elevation can be slightly different from the harmonic profile required. In order to provide the most accurate comparison of model performance, the PoT models take the actual wave profile, measured in CFD, as an input.

Real wave energy applications may benefit from the implementation of a controller, which magnifies the amplitude of motion, in order to increase power absorption. For the purpose of this paper, latching control has been chosen, since it is a simple discrete strategy, particularly able to induce high velocities and therefore likely to reveal differences between various modelling approaches. Results are compared with the uncontrolled case (resistive damping PTO), in order to highlight how nonlinearities are excited by the action of the controller.

Latching control requires the definition of only two parameters, namely the PTO damping coefficient $B_{p t o}$ and the latching duration $D_{L}$. The same PTO damping has been chosen for all models, set equal to the radiation damping $B(\omega)$, at the wave frequency $\omega_{w}$, according to the linear optimal energy absorption condition (Falnes et al., 2002). The zero-threshold criterion (Falcao, 2008) is applied for the release instant selection, following a zero-crossing in the excitation force. Note that, still following the objective of establishing a level field of comparison, a linear excitation force is considered for all the models, so that the release time is the very same for each model. 


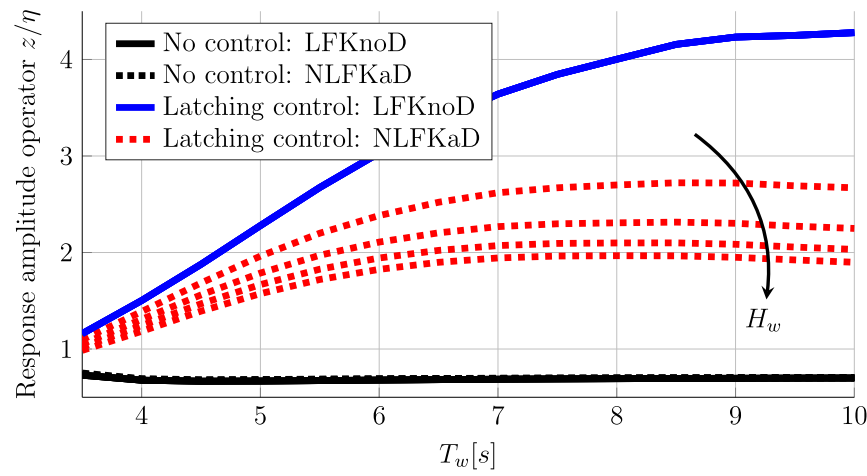

Fig. 6. Response amplitude operator, with and without latching control, considering LFKnoD and NLFKaD models.

As a remark, note that the objective is not to optimize the power capture, but rather to have an identical control strategy for each model configuration. Indeed, Giorgi and Ringwood (2016b) shows how the optimal PTO damping coefficient and latching duration are significantly different, when nonlinearities are included.

\section{Results}

The response amplitude operator is studied, with and without latching control applied, at different $T_{w}$ and $H_{w}$, comparing the (fully-linear) LFKnoD model to the NLFKaD model, as shown in Fig. 6. On the one hand, without control, LFKnoD and NLFKaD curves overlap, due to the negligible impact of FK and viscous nonlinearities. In fact, the uncontrolled RAO is close to unity, and almost constant, so the floating sphere tends to behave as a wave follower, with a consequent small relative motion with respect to the free surface elevation.

On the other hand, the latching control strategy exaggerates the amplitude of motion, increasing the relevance of FK and viscous nonlinearities. Consequently, the fully-linear model significantly overestimates the response of the device. Furthermore, the NLFKaD model is represented by different curves at different $H_{w}$, decreasing as $H_{w}$ increases, highlighting that such nonlinear effects become more important as the wave energy content increases.

The accuracy of the results obtained with the PoT models is evaluated against the CFD results. Considering one period of the steady state response, an error index is defined for each model $j$ through the normalized root mean squared error of the vertical displacement $z_{j}$, as follows:

$e_{j}=\frac{1}{\overline{z_{C F D}}} \sqrt{\frac{\sum_{t_{i}=t_{0}}^{t_{0}+T_{w}}\left(z_{C F D}\left(t_{i}\right)-z_{j}\left(t_{i}\right)\right)^{2}}{n}}$

where $n$ is the number of time samples and $\overline{z_{C F D}}$ is the difference between the maximum and minimum values of the displacement in the CFD model.

Fig. 7 shows the error indeces for all waves and models, with latching control. For each wave period and height, six sets of bars are grouped together, three in the horizontal direction and two in the vertical direction. The three bar sets represent the FK force modelling options, respectively, from left to right: linear (LFK), nonlinear restoring (NLR), and nonlinear FK force (NLFK). Note that the two modelling options for the nonlinear FK force (re-meshing (NLFKr) and algebraic (NLFKa) approaches) return exactly the same results, with different computational times, so they are represented with the same bar, for brevity. For each FK force modelling option, the upper bar is without drag (noD), whereas the lower one includes (D). In interpreting the results shown in Fig. 7, consider that the final total accuracy is a consequence of several interacting modelling errors, which may add up or cancel out, as discussed in Section 2.1.
Overall, it is clear that the inclusion of viscous drag in the model significantly reduces the error in the resulting estimation of displacements, no matter what FK force modelling option is adopted. In general, when viscous drag is implemented, errors are quite low for all the wave conditions considered. Furthermore, when drag is modelled, the differences between different FK force models is also relatively small. The reason for this reduction in model difference is that viscous drag is restraining the device motion to such an extent that the variations in instantaneous wetted surface are limited, making nonlinear FK force modelling less relevant.

Conversely, when drag is not implemented, considerable differences between FK force models (and larger errors) may arise for more energetic waves (large periods and/or heights). Furthermore, since the nonlinear restoring force model (NLR) loses the mitigation effect described in Section 2, larger errors are consistently obtained, compared to the linear model. Overall, the accuracy of the results is largely attributed to the implementation of the FK force only in the absence of viscous damping, and under controlled conditions. A more detailed discussion about the reasons why viscous drag is more important than nonlinear FK force, and the way in which the dynamics of the device are changed by such nonlinearities, will be presented in Section 5 .

As in Figs. 7 and 8 shows the error indeces without control applied for all the wave conditions considered. Comparing Fig. 8 with Fig. 7, it is clear that lower errors are obtained in the uncontrolled case. Furthermore, differences between the models are negligible since, as expected, the absence of a control strategy results in small relative motion, with consequent weak nonlinearities. Consequently, for the uncontrolled case, linear models return approximately the same results as nonlinear models, but with a lower computational price. Section 5 shows how the control strategy prevents the device from behave as a wave follower since, in the absence of control, relative body/fluid displacement and velocity are small.

The error index is not the only quality indicator to evaluate the performance of the models. Including nonlinearities in the model has a complexity and computational cost. Ultimately, the computational speed of a model has to be evaluated with respect to real time; therefore, the ratio between the computational time $t_{\text {comp }}$, and the simulated time $t$, is considered.

For PoT models, a second order Runge-Kutta scheme is implemented in MATLAB on a machine with an Intel(R) Xeon(R) CPU (E5-1620 v3@ $3.50 \mathrm{GHz}$ ) processor, with $16.0 \mathrm{~GB}$ RAM and Windows 7 Professional 64 bit.

The CFD calculations are performed on a high-performance computing cluster made available by the Irish Center for High-End Computing (ICHEC) (ICHEC, 2016). Each core, of the machines in ICHEC, carries an Intel Xeon E5-2695 2.4 GHz processor and several nodes can be used, each one with 24 cores. For the purpose of this paper, simulations used from 24 to 192 cores, according to the computational domain and simulation time, in order to meet the $72 \mathrm{~h}$ time limit imposed by ICHEC. In order to have a fair comparison, the computational time considered has been scaled linearly as if only one core was used, therefore introducing an up-scaling factor of about 1-2 order of magnitudes, between 24 and 192. Note that an error is introduced since, in general, the computational time does not scale linearly with the number of processors used. The time ratio-error index couples, for the nine models and 24 wave conditions considered, are plotted in Fig. 9.

Each point is identified by two concentric markers: the outer marker indicates which of the four FK force modelling options is used, while the inner one indicates the presence/absence of viscous drag. The cloud of points for each model is fitted with an ellipse, in order to define a clear region in the graph. Since, as the reference model, the error for the CFD model is zero, by definition, it is represented with a line, and no markers are used.

As expected, the CFD computational time is several orders of magnitude longer than the other models, requiring from $10^{5}$ to $10^{6} \mathrm{~s}$ 

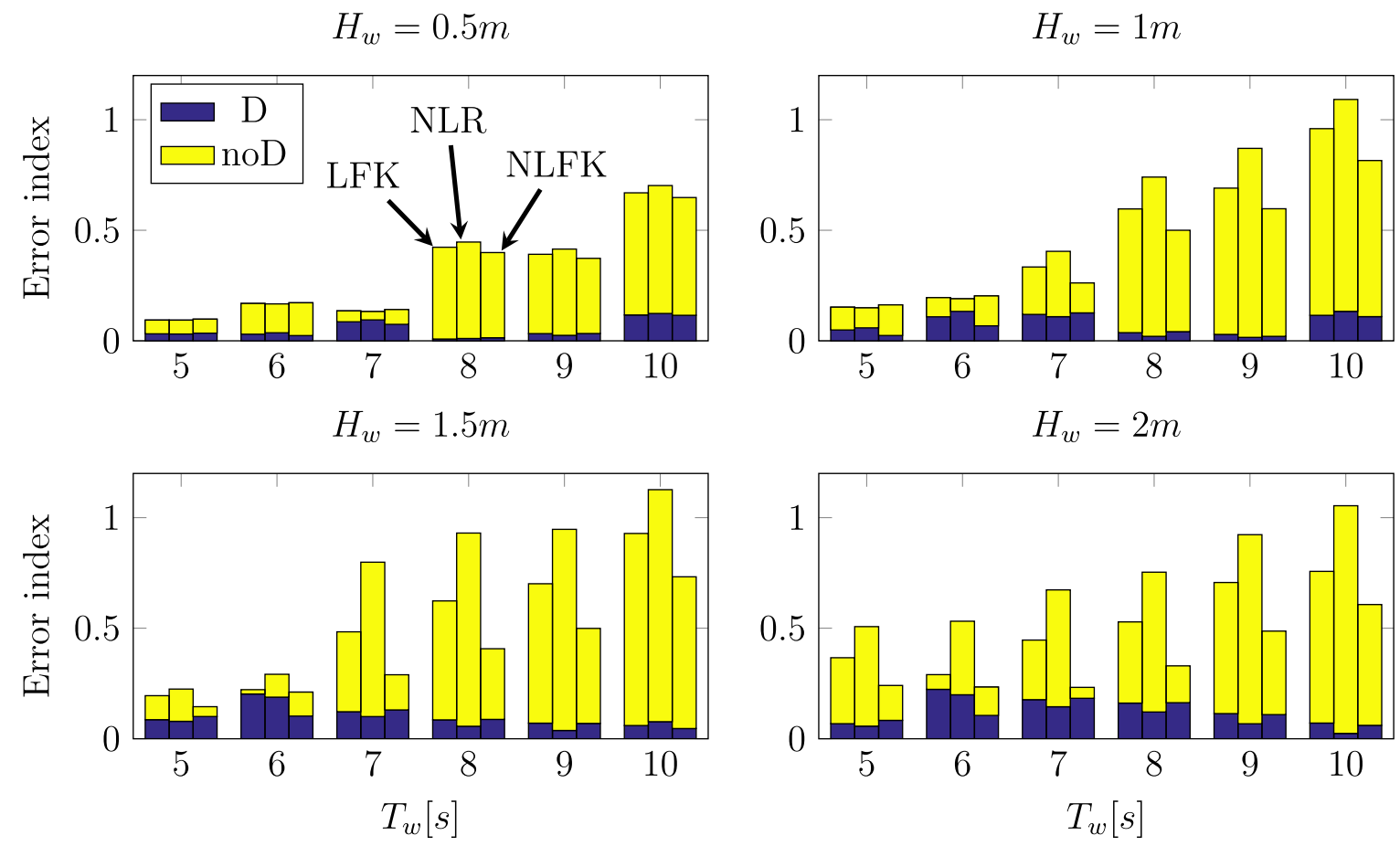

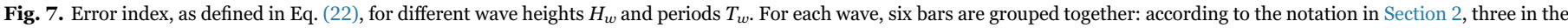

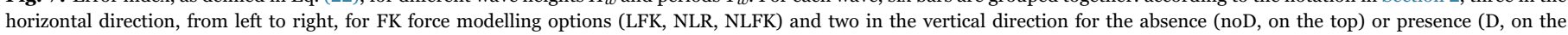
bottom) of viscous drag in the model.

to perform one second of simulation (using the same comparative hardware capabilities as the other models). As a result of the logarithmic scale of the horizontal axis, the PoT models are squeezed to the left hand side of Fig. 9.

Consistent with Figs. 7 and 9 demonstrates that errors without drag are much larger than when viscous drag is included. Furthermore, errors in the linear model are smaller than in the nonlinear restoring force model, but larger than in the nonlinear FK force model. With regard to the computation time ratio, the nonlinear restoring force and
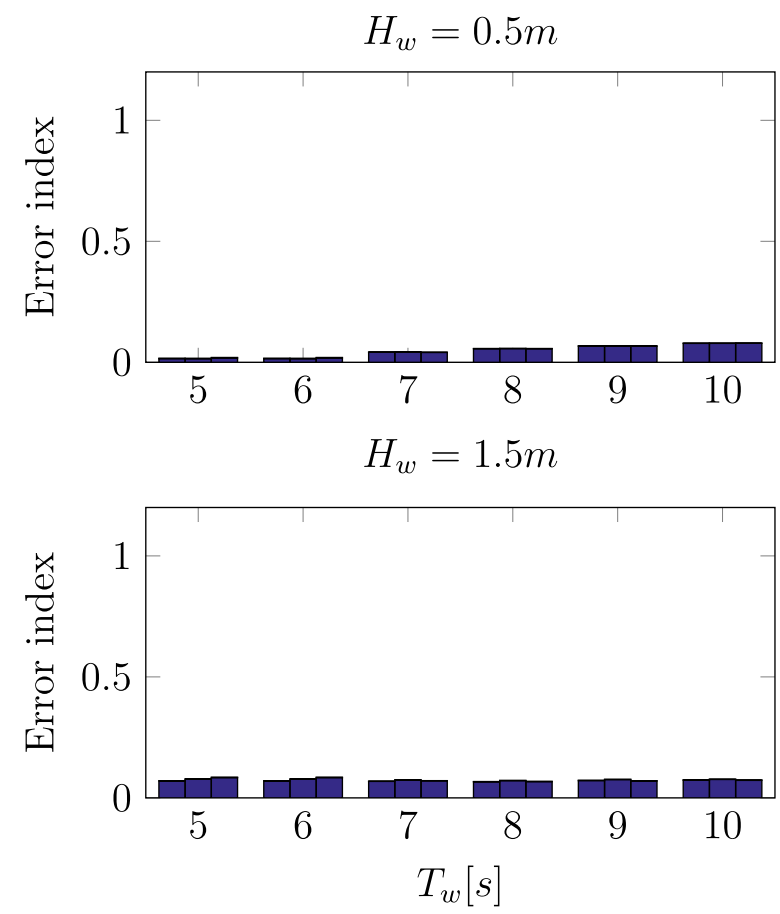

algebraic nonlinear FK force models are up to an order of magnitude slower than the linear models. On the other hand, the re-meshing approach model (NLFKr) returns identical errors to the algebraic model (NLFKa), but at computational time almost an order of magnitude larger. Finally, the inclusion of viscous drag increases the computational time of each of the four FK models, but in a more pronounced way for the linear model, than for the others. The mean value and the relative standard deviation for the computational times of each PoT model have been summarized in Table 2.

$$
H_{w}=1 m
$$
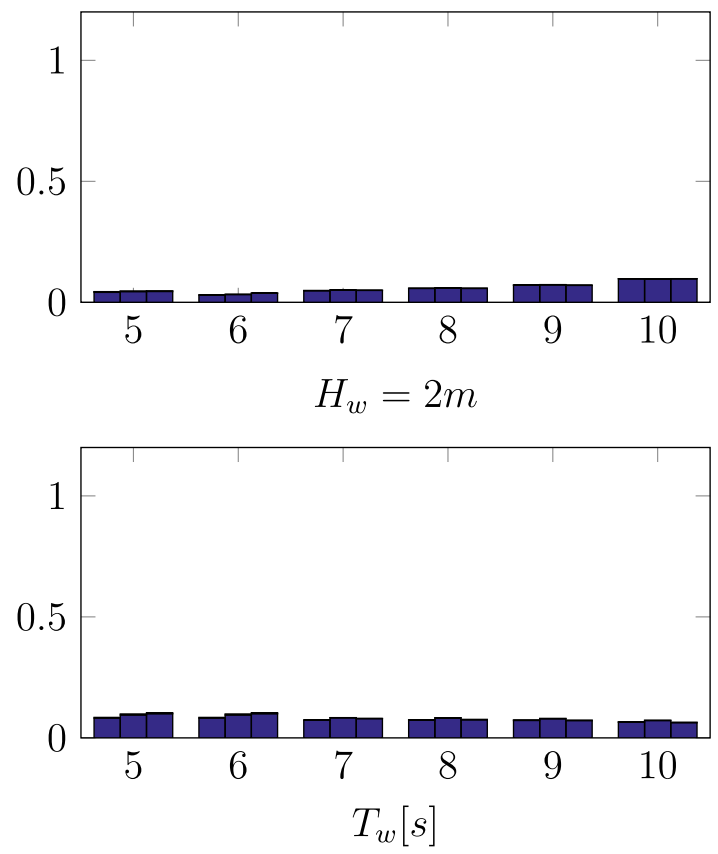

Fig. 8. Error index as in Fig. 7, but without latching control. 


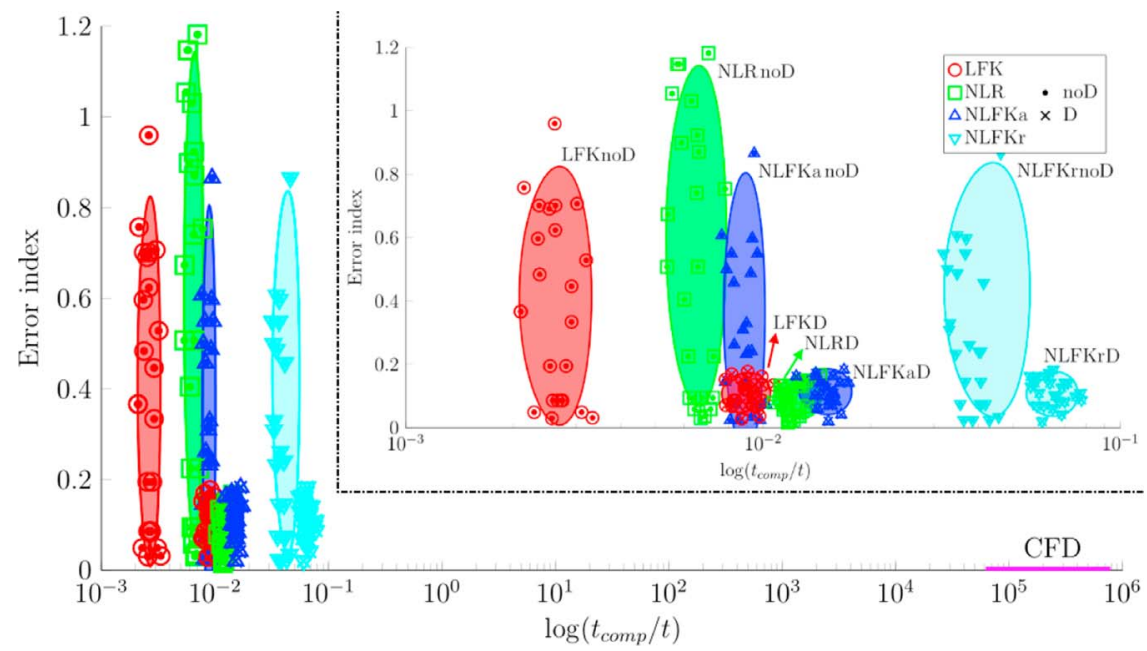

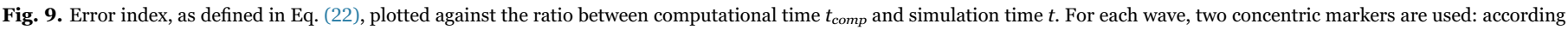

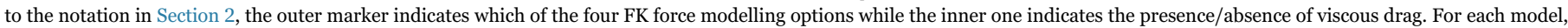
the cloud of points has been fitted with an ellipse.

Table 2

Mean and relative standard deviation of computational time for each PoT model, as shown in Fig. 9.

\begin{tabular}{lll}
\hline $\log \left(t_{\text {comp }} / t\right)$ & Mean $10^{-3}$ & Relative standard deviation \\
\hline LFKnoD & 2.63 & $12.7 \%$ \\
NLRnoD & 6.43 & $9.7 \%$ \\
NLFKanoD & 8.90 & $7.9 \%$ \\
NLFKrnoD & 38.1 & $10.4 \%$ \\
LFKD & 9.06 & $8.1 \%$ \\
NLRD & 12.1 & $6.9 \%$ \\
NLFKaD & 15.5 & $7.1 \%$ \\
NLFKrD & 65.0 & $9.8 \%$ \\
\hline
\end{tabular}

\section{Discussion}

The relevance of FK and viscous nonlinearities, for the floating sphere considered in this study, depends mainly on the amplitude of the device motion with respect to the free surface elevation. In the uncontrolled case, small relative motions around the free surface are obtained, with resulting low impact of FK and viscous nonlinearities. Consequently, nonlinearities are enhanced by the control strategy, whose main effect is to considerably enlarge the relative motion between the floater and the free surface. In particular, when no control is applied, the device behaves as a wave follower, where the relative displacement is not significant. Fig. 10 shows a graphical representation of the device motion obtained for the CFD case, with and without control applied: Fig. 10(a) and (b) show, respectively, the peak and bottom position of the floater without control, while Fig. 10(c) and (d) show, respectively, the peak and bottom position at the unlatching instant. Without control, the center of the body is approximately at the free surface elevation height; therefore, the floater behaves as a wave follower, and nonlinearities are negligible. On the contrary, when control is applied, a larger amplitude of motion, and significant variations in the instantaneous wetted surface, arise.

Fig. 11 shows the complete operational space (relative displacement and relative velocity) of the steady state response of the device, for an example of a regular wave, with and without control applied. Nonlinearities in FK forces depend on the variation in the instantaneous wetted surface, and therefore on the relative displacement between the device and the free surface elevation. Likewise, viscous drag forces have a quadratic dependence on the relative velocity.

It is clear that the action of the controller considerably enlarges the operational space and, consequently, the relevance of nonlinearities.
Indeed, only one curve is presented for the uncontrolled case, since the response is largely the same, irrespective of which model is used.

On the other hand, the fully-linear and fully-nonlinear models, which are presented for the controlled case as two extreme representative implementations, show large differences in the simulated response, highlighting how nonlinearities are enhanced by the controller.

However, the device is normally working under controlled conditions, since the objective is to increase power absorption. Indeed, one of the most fundamental outputs of a simulation is the estimation of power production. Under controlled conditions, neglecting to model viscous drag effects may cause a significant overestimation of power production, leading to overly optimistic expectations. Fig. 12 shows the ratio between the power estimation according to the linear and the fully-nonlinear models, while Table 3 tabulates the ratio for all the models. The ratio presented in Fig. 12 varies between 1 and 4.45, and the overall trend is increasing with both wave height and period, as the more energetic waves cause larger oscillations. The translucent plane in Fig. 12 corresponds to a unity power ratio, which is the fidelity benchmark.

Overall, Table 3 confirms the trend shown in Figs. 7 and 12 . Additional information, that is clear from Table 3, is that the ratio for the models with drag, especially those with additional nonlinear FK force, are relatively low (which is good) but quite variable and can be even less than unity. Such a trend suggests that the drag coefficient, which would allow the best fit with the CFD model and, consequently, ensure a unity power ratio, is variable and lower than the drag coefficient chosen according to Babarit et al. (2012).

It is indeed possible to use the results from CFD simulations to estimate the best drag coefficient for each wave condition; Giorgi and Ringwood (2017a) uses a least squared method approach to identify the drag coefficient which minimizes the error between the NLFKaD PoT model and the CFD model. The resulting $C_{d}$ is tabulated in Table 4. As expected, the optimal $C_{d}$ is overall variable, and lower than unity for almost all the wave conditions considered. Nevertheless, note that such a viscous term in the Morison equation would tend to try to describe not only the viscous drag term, but also other residual nonlinearities (such as nonlinear diffraction, nonlinear radiation, or non-Airy's dynamical pressure distribution), which are not modelled in PoT models, but are present in the fully-nonlinear CFD model. In fact, Giorgi and Ringwood (2017a) refers to the optimal $C_{d}$, in Table 4, as an "equivalent" $C_{d}$, since it actually includes different sources of nonlinearity, apart from viscous drag. Consequently, KC (between 0.56 and 3.11) and $\operatorname{Re}$ (between $3.56 \mathrm{e} 6$ and 1.79e7), calculated from CFD 


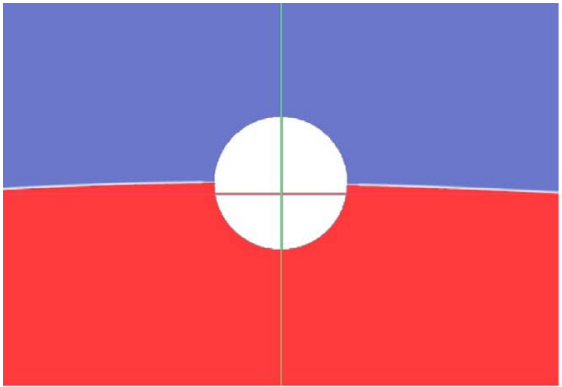

(a) Top position without control

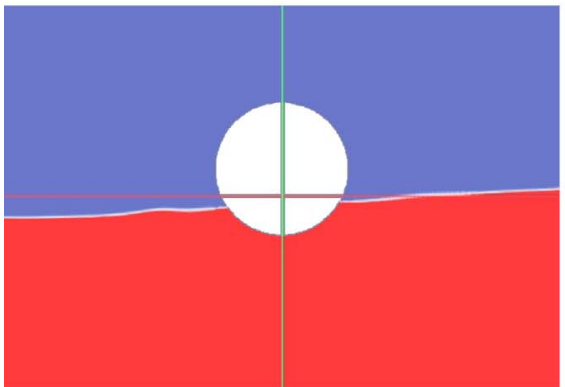

(c) Top position with control at (d) Bottom position with control the unlatching instant.

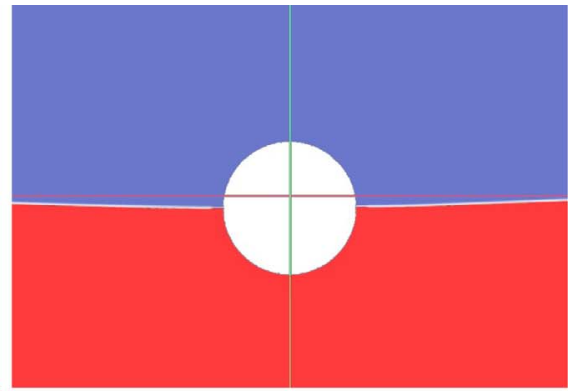

(b) Bottom position without control.

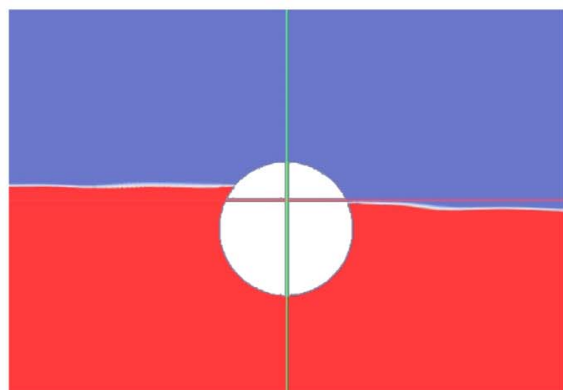

Fig. 10. Screen-shots of CFD simulations with and without control applied (Giorgi et al., 2016b).

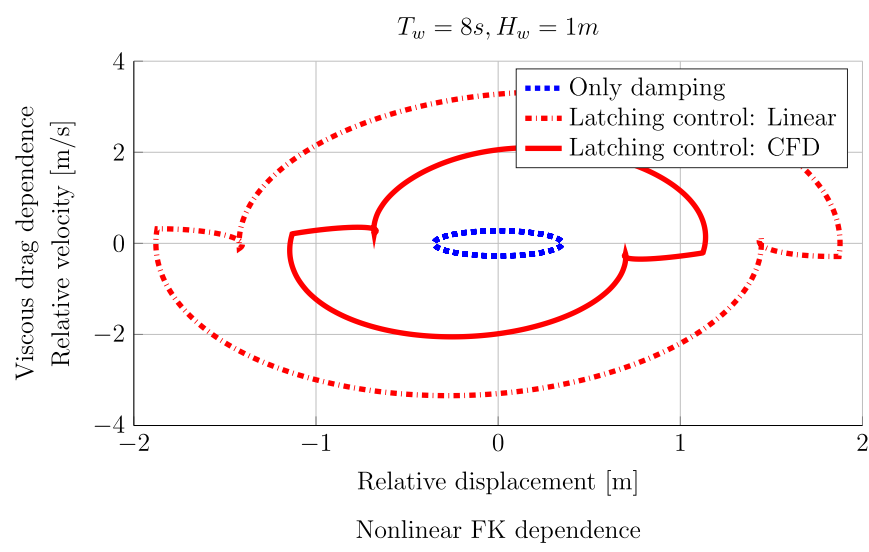

Fig. 11. Steady state operational space for a regular wave with period $T_{w}=8 \mathrm{~s}$ and height $H_{w}=1 \mathrm{~m}$. For the uncontrolled case (PTO acting as a simple damper), only one curve is shown since all models substantially overlap. Conversely, under controlled conditions, the fully-linear and fully-nonlinear responses are significantly different and enlarged with respect to the uncontrolled case.

simulations, are not full explanatory of the "equivalent" $C_{d}$ (Giorgi and Ringwood, 2017a), nor justify its variability.

A closer look on how nonlinear FK and viscous drag forces influence the dynamics of the device can give further insight into the relevance of each nonlinearity, and the way they interact with each other. Fig. 13 shows nonlinear FK and viscous drag forces, for one period of the periodic response, under the action of one sample regular wave. The relative displacement is presented on a second vertical axes, in order to evaluate the phase between the response and the forces.

In order to highlight the differences due to modelling only, the linear FK force calculation has been applied to the operational space given by the nonlinear model. Finally, the latching interval is shaded (in red), to indicate that external hydrodynamic forces actually have no

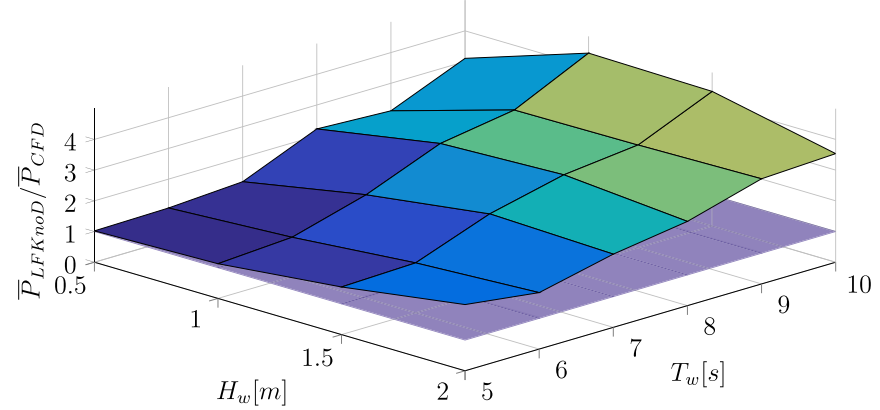

Fig. 12. Ratio between the predicted power outputs according to the fully-linear model (LFKnoD) and the fully-nonlinear model(CFD). The translucent plane indicates the unity ratio, which corresponds to maximum fidelity.

influence on the device motion, since the latching mechanism is preventing any body motion. Such intervals are those where the largest relative displacement and, consequently, the largest differences between linear and nonlinear FK forces are found. Conversely, the device velocity is zero, with a correspondingly null viscous drag force.

On the other hand, the regions where the device is free to move, and therefore sensitive to modelling errors, are the regions where the highest velocities and smallest relative displacements are achieved. As a consequence, differences between linear and nonlinear FK forces are smaller, whereas viscous drag, which is completely absent in the linear model, has its strongest influence on the device.

An alternative way of analysing how each force component influences the system's dynamics is to consider its instantaneous relative importance; at every time instant, a force ratio is defined as the fraction between the absolute value of the particular force component and the sum of the absolute values of all the force components on the right hand side of the equation of motion (8). Absolute values are used in order to have the ratios bounded between 0 and 1 ; otherwise, due to 
Table 3

Ratio between the predicted power outputs according to each of the potential theory-based models and the fully-nonlinear (CFD) model.

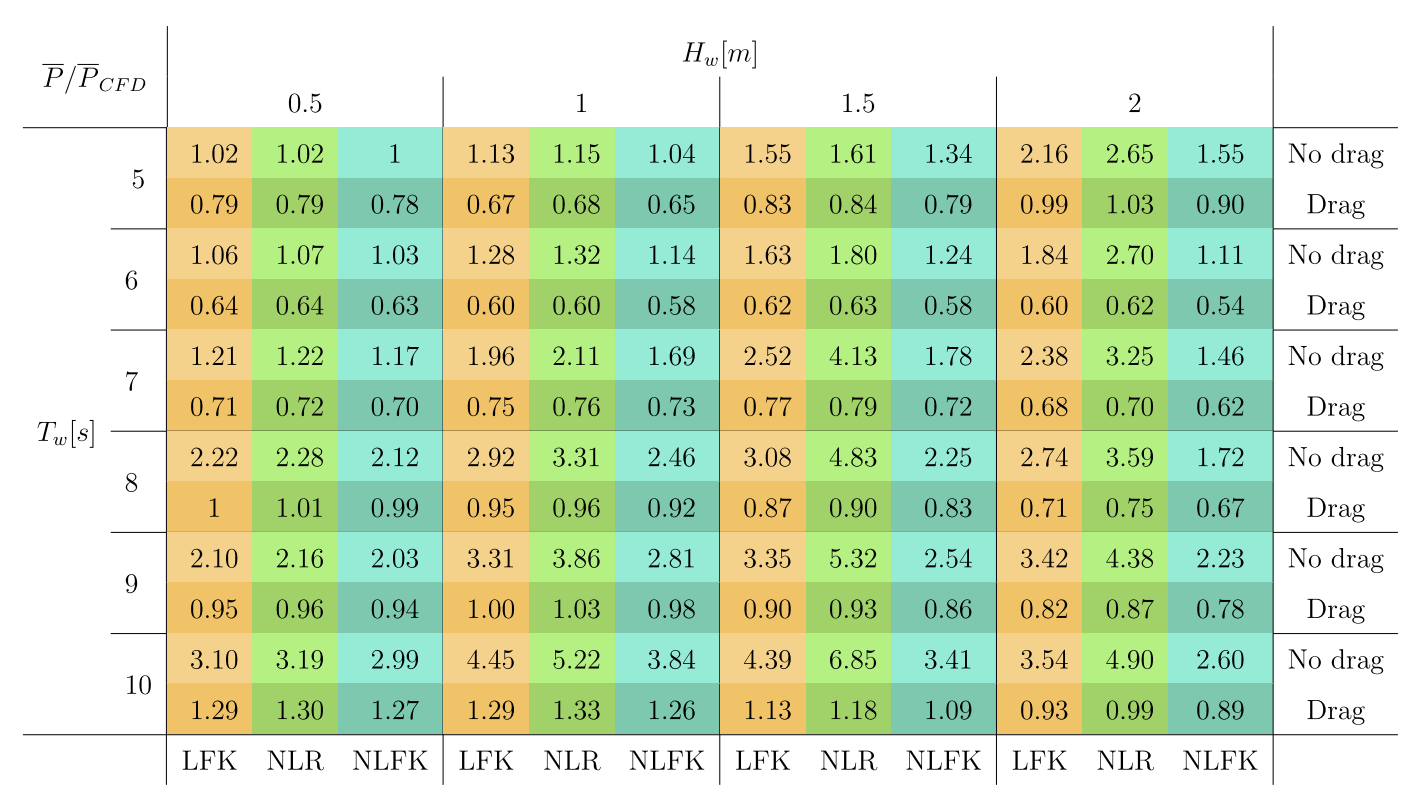

\section{Table 4}

Estimated drag coefficients, which minimize the least square error between the NLFKaD model and the CFD model, under latching control conditions (Giorgi and Ringwood, 2017a).

\begin{tabular}{|c|c|c|c|c|c|}
\hline \multirow[b]{2}{*}{$C_{d}$} & & \multicolumn{4}{|c|}{$H_{w}[\mathrm{~m}]$} \\
\hline & & 0.5 & 1 & 1.5 & 2 \\
\hline \multirow[t]{6}{*}{$T_{w}[s]$} & 5 & 0.05 & 0.05 & 0.33 & 0.47 \\
\hline & 6 & 0.06 & 0.13 & 0.18 & 0.19 \\
\hline & 7 & 0.29 & 0.46 & 0.49 & 0.33 \\
\hline & 8 & 0.97 & 0.81 & 0.66 & 0.54 \\
\hline & 9 & 0.88 & 0.89 & 0.73 & 0.59 \\
\hline & 10 & 1.34 & 1.43 & 1.15 & 0.79 \\
\hline
\end{tabular}

phase differences, each ratio could be larger than 1, positive or negative. Fig. 14 shows the FK and viscous drag ratios, for regular waves with $T_{w} 8 \mathrm{~s}$, for four different values of $H_{w}$, where the FK ratio is

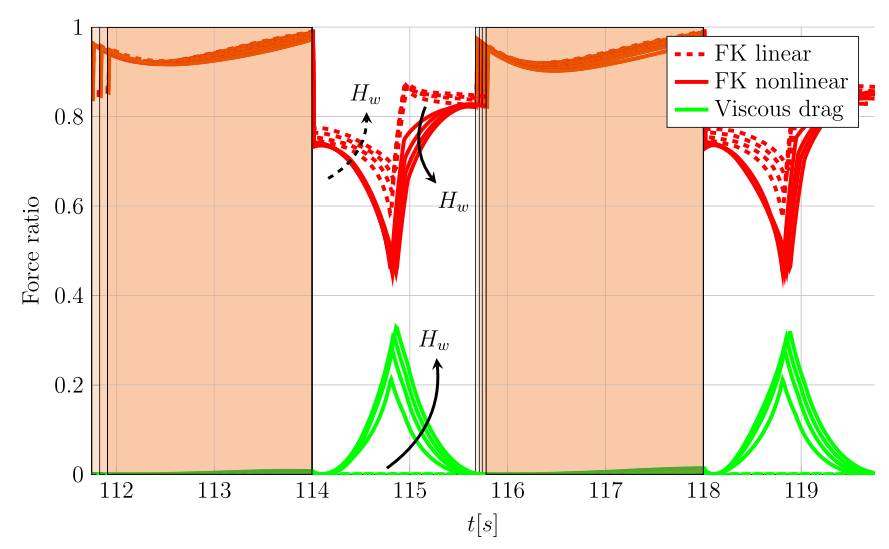

Fig. 14. Froude-Krylov and viscous drag force ratios for a wave with $T_{w} 8 \mathrm{~s}$ and four $H_{w}$, according to NLFKaD model. Linear force calculations have been applied to displacement and velocity obtained with the nonlinear model in order to compare modelling differences only.

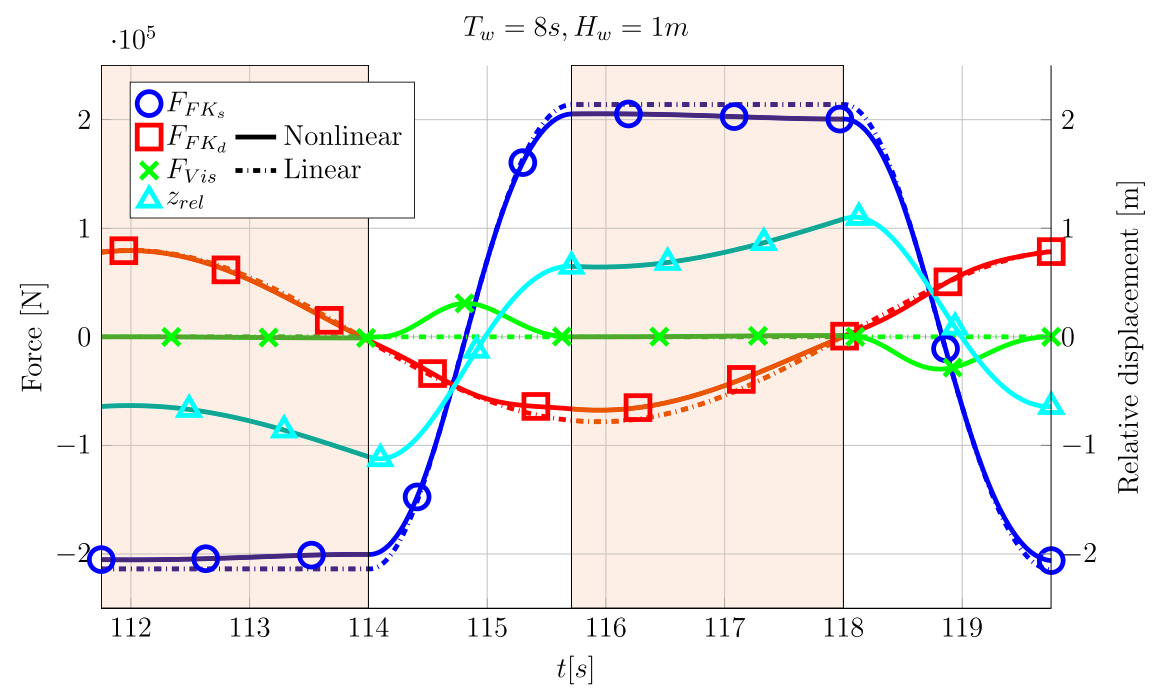

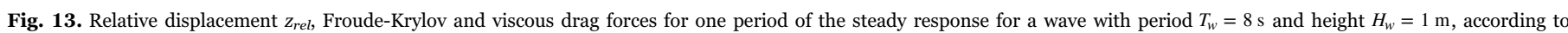
NLFKaD model. Linear force calculations have been applied to displacement and velocity obtained with the nonlinear model in order to compare modelling differences only. 


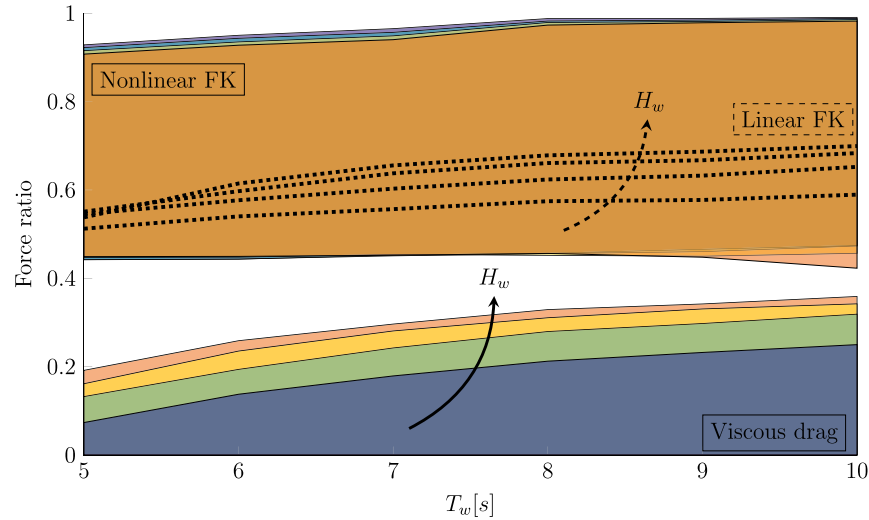

Fig. 15. Ranges of variation (from minimum to maximum) of Froude-Krylov and viscous drag ratios.

the sum of the static FK ratio and dynamic FK ratio. As in Fig. 13, the latched intervals are shaded (in red).

Considering the free response interval (where the body is not latched and free to move), as the viscous drag ratio increases (due to increasing velocities), the $\mathrm{FK}$ ratio decreases. Moreover, as $H_{w}$ increases, the viscous drag ratio increases, making the FK ratio decrease as a consequence. In contrast, the linear FK ratio increases with $H_{w}$, since viscous drag is absent in the fully-linear model.

Fig. 14 shows the continuous variations of the different force ratios throughout one period of motion. In order to have a more global view, the ranges of variation (from minimum to maximum) of such ratios are shown in Fig. 15, for all the sea conditions considered in this paper. Note that the minimum FK ratio occurs at the maximum of the drag ratio and, likewise, the maximum FK ratio (almost 100\%) occurs at the minimum of the drag ratio (equal to zero).

The maximum viscous drag ratios and the minimum linear FK ratios increase as $H_{w}$ increases, whereas the nonlinear FK ratio bounds remain relatively constant. Furthermore, the difference between the linear and nonlinear representation of FK forces is significant only at the lower bound, varying from a minimum of $9 \%$ (at $T_{w} 5 \mathrm{~s}, H_{w} 0.5 \mathrm{~m}$ ), to a maximum of $28 \%$ (at $T_{w} 10 \mathrm{~s}, H_{w} 2 \mathrm{~m}$ ).

As a final remark, consider that the FK ratio, plotted in Figs. 14 and 15 , is the sum of the absolute values of the static and dynamic FK ratios, in order to be consistent with the equation of motion in (8). Furthermore, such a choice enhances the clarity and readability of Figs. 14 and 15 more clear and easy to read. Consider now an interchange of the order of summation and absolute value: first compute the total FK force, as the sum (with sign) of its static and dynamic components, and afterwards take the absolute value. Since $F_{F K_{s}}$ and $F_{F K_{d}}$ are not in phase (Giorgi and Ringwood, 2017b), they partially cancel out. Consequently, the minimum values of the total FK force ratio drop drastically, reaching values of about 1-2\%. Likewise, the maximum viscous drag ratios considerably increase, between 1.8 and 2.5 times the values shown in Fig. 15.

\section{Conclusions}

The choice of an appropriate mathematical model for wave energy converters is not straightforward and needs to define the right compromise of accuracy and computational cost to satisfy the specific requirements of the application the model is intended for.

This paper analyzes the performance of nine mathematical models, when a device is operating in the power production region, eight of which are based on potential theory, with different modelling options for FK and viscous drag forces, while the ninth model is based on CFD. The models have been evaluated according to accuracy and computational time indeces.

Overall, in can be broadly concluded that Froude-Krylov and viscous drag nonlinearities are insignificant for uncontrolled small heaving floating devices, operating at wave periods away from resonance; therefore, linear models are to be preferred. Nevertheless, a control strategy is likely to be included in a real WEC application, since the very objective of a wave energy converter is to maximize power absorption.

Results suggest that, under latching control conditions, viscous forces are large enough to reduce the body's displacement to such an extent that a linear description of FK forces becomes quite accurate. Nevertheless, the drag coefficient appears to be overestimated in this study, and varies across the considered wave conditions; therefore, the identification of a single ideal value can be challenging.

In general, accurate models do not necessarily imply accurate results, and vice versa. In fact, multiple sources of modelling errors interact, in a constructive or destructive way, in order to produce the total error - and accuracy - of the model. In particular, it is found, in this study, that the modelling errors due to the linear representation of both FFKs and FFKd partially cancel out (LFK model), so that the total error is lower than the case where only a FK modelling error is present (NLR model), but greater than the case where no FK modelling error is present (NLFK model).

Secondly, the constant (overestimated) drag coefficient introduces a modelling error (and uncertainty) in the viscous force representation; nevertheless, the resulting accuracy may potentially improve, since the erroneous viscous drag coefficient may cover the effect of other modelling errors and unmodelled nonlinearities.

Furthermore, it is found that viscous drag is particularly important for a floating device under latching control conditions. In fact, FK forces are dominant only in intervals where the device is latched, therefore constrained not to move. On the contrary, when the body is released and responds to external forces, the relevance of viscous forces is enhanced by large velocities and small (relative) displacements.

One of the limitations of the study is the degree to which a CFD model can be considered a gold standard. There is an alternative view that tank tests, or open ocean measurements, provide a better reference point. However, tank tests or open ocean tests also suffer from difficulties, viz. tank reflections, and quantification of the incident wave field, respectively. At the very least, it is a worthwhile endeavour to attempt to synthesise a model with a fidelity close to that of CFD, at a fraction of the computational cost.

\section{Acknowledgment}

This paper is based upon work supported by Science Foundation Ireland under Grant No. 13/IA/1886. The authors acknowledge as well the support of the Irish Centre for High-End Computing (ICHEC).

\section{References}

Babarit, A., Hals, J., Muliawan, M., Kurniawan, A., Moan, T., Krokstad, J., 2012. Numerical benchmarking study of a selection of wave energy converters. Renew. Energy 41, 44-63.

Bhinder, M.A., Babarit, A., Gentaz, L., Ferrant, P., 2011. Assessment of viscous damping via $3 d$-cfd modelling of a floating wave energy device. In: Proceedings of the 9th European Wave and Tidal Energy Conference. Southampton, UK.

Falcao, A., 2008. Phase control through load control of oscillating-body wave energy converters with hydraulic pto system. Ocean Eng. 35 (3), 358-366.

Falnes, J., 2002. Ocean Waves and Oscillating Systems. Cambridge University Press, Cambridge, UK.

Ferziger, J.H., Peric, M., Leonard, A., 1997. Computational Methods for Fluid Dynamics.

Forehand, D.I., Kiprakis, A.E., Nambiar, A.J., Wallace, A.R., 2016. A fully coupled waveto-wire model of an array of wave energy converters. IEEE Trans. Sustain. Energy 7 (1), 118-128.

Gilloteaux, J.-C., 2007. Mouvements de grande amplitude d'un corps flottant en fluide parfait. application à la récupération de l'énergie des vagues (Ph.D. thesis). Ecole Centrale de Nantes-ECN.

Giorgi, G., Ringwood, J.V., 2016a. Implementation of latching control in a numerical wave tank with regular waves. J. Ocean Eng. Mar. Energy 2 (2), 211-226.

Giorgi, G., Ringwood, J.V., 2016b. Computationally efficient nonlinear froude-krylov force calculations for heaving axisymmetric wave energy point absorbers. J. Ocean Eng. Mar. Energy, 1-13. 
Giorgi, G., Penalba, M., Ringwood, J.V., 2016a. Nonlinear hydrodynamic force relevance for different wave energy converter types. In: Proceedings of the 3rd Asian Wave and Tidal Energy Conference. pp. 154-162.

Giorgi, G., Penalba, M., Ringwood, J.V., 2016b. Nonlinear hydrodynamic models for heaving buoy wave energy converters. In: Proceedings of the 3rd Asian Wave and Tidal Energy Conference, pp. 144-153.

Giorgi, G., Ringwood, J.V., 2017a. Consistency of viscous drag identification tests for wave energy applications. In: Proceedings of the 12th European Wave and Tidal Energy Conference (EWTEC). Cork.

Giorgi, G., Ringwood, J.V., 2017b. Comparing nonlinear hydrodynamic forces in heaving point absorbers and oscillating wave surge converters. J. Ocean Eng. Mar. Energy, Submitted for publication.

ICHEC, Irish Centre for High-End Computing (ICHEC), 2016.URL 〈https://www.ichec. ie/ $/$.

Jacobsen, N.G., Fuhrman, D.R., Fredsoe, J., 2012. A wave generation toolbox for the open-source cfd ligrary: Openfoam. Int. J. Numer. Methods Fluids 70, 1073-1088.

Keulegan, G.H., Carpenter, L.H., 1956. Forces on Cylinders and Plates in an Oscillating Fluid. US Department of Commerce, National Bureau of Standards.

Lok, K., Stallard, T., Stansby, P., Jenkins, N., 2014. Optimisation of a clutch-rectified power take off system for a heaving wave energy device in irregular waves with experimental comparison. Int. J. Mar. Energy 8, 1-16.

Merigaud, A., Gilloteaux, J.-C., Ringwood, J.V., 2012. A nonlinear extension for linear boundary element methods in wave energy device modelling. In: Proceedings of the 31st International Conference on Ocean, Offshore and Arctic Engineering, American Society of Mechanical Engineers (ASME 2012). pp. 615-621.

Molin, B., 2002. Hydrodynamique des structures offshore. Editions Technip.

Morison, J.R., Johnson, J.W., Schaaf, S.A., 1950. The force exerted by surface waves on piles. J. Pet. Technol. 2 (5), 149-154.

Newman, J., 1977. Marine Hydrodynamics. MIT Press, Cambridge, UK.

OpenFOAM. OpenFOAM The Open Source CFD Toolbox User Guide Version 3.0.1 (13th December 2015)

Penalba, M., Giorgi, G., Ringwood, J.V., 2015. A review of non-linear approaches for wave energy converter modelling. In: Proceedings of European Wave and Tidal Energy Conference. Nantes, France.

Penalba, M., Merigaud, A., Gilloteaux, J.C., Ringwood, J.V., 2015. Nonlinear FroudeKrylov force modelling for two heaving wave energy point absorbers. In: Proceedings of European Wave and Tidal Energy Conference, Nantes, France.

Taghipour, R., Perez, T., Moan, T., 2007. Hybrid frequency-time domain models for dynamic response analysis of marine structures. Ocean Eng. 35 (7), 685-705.

Temam, R., 2001. Navier-Stokes Equations: Theory and Numerical Analysis 343. American Mathematical Soc, Amsterdam, Netherlands.

W. Inc., WAMIT v7.0 manual, 2013.

Wavestar, Wavestar a/s, 2016. Available at $\langle$ http://wavestarenergy.com/ $\rangle$. 